\title{
Measurements of Droplet Size in Shear-Driven Atomization Using Ultra- Small Angle X-Ray Scattering
}

\author{
A. Kastengren $^{a^{*}}$, J. Ilavsky ${ }^{\mathrm{a}}$, Juan Pablo Viera ${ }^{\mathrm{b}}$, Raul Payri $^{\mathrm{b}}$, D. J. Duke ${ }^{\mathrm{c}}$, A. Swantek ${ }^{\mathrm{c}}$, F. Zak \\ Tilocco $^{\text {c1 }}$, N. Sovis ${ }^{\mathrm{c}}$, and C.F. Powell ${ }^{\mathrm{c}}$ \\ ${ }^{a}$ X-Ray Science Division, Advanced Photon Source, Argonne National Laboratory, 9700 S. Cass \\ Ave, Argonne IL 60439 USA \\ ${ }^{\mathrm{b}} \mathrm{CMT}$-Motores Térmicos, Universitat Politècnica de València, Spain \\ ${ }^{c}$ Energy Systems Division, Argonne National Laboratory, 9700 S. Cass Ave, Argonne IL 60439 \\ USA \\ *Corresponding author, akastengren@anl.gov \\ ${ }^{1}$ Present address: Michigan State Univeristy, East Lansing, MI USA
}




\section{Abstract}

Measurements of droplet size in optically-thick, non-evaporating, shear-driven sprays have been made using ultra-small angle x-ray scattering (USAXS). The sprays are produced by orifice-type nozzles coupled to diesel injectors, with measurements conducted from $1-24 \mathrm{~mm}$ from the orifice, spanning from the optically-dense near-nozzle region to more dilute regions where optical diagnostics are feasible. The influence of nozzle diameter, liquid injection pressure, and ambient density were examined. The USAXS measurements reveal few if any nanoscale droplets, in conflict with a popular computational model of diesel spray breakup. The average droplet diameter rapidly decreases with downstream distance from the nozzle until a plateau value is reached, after which only small changes are seen in droplet diameter. This plateau droplet size is consistent with the droplets being small enough to be stable with respect to further breakup. Liquid injection pressure and nozzle diameter have the biggest impact on droplet size, while ambient density has a smaller effect.

\section{Keywords}

Diesel spray, droplet size, x-ray scattering

\section{Introduction}

Liquid spray flowfields are of significant interest for both applied and fundamental studies. They are crucial to the mixing of gases and liquids, and of particular importance in liquid-fueled combustion. From a fundamental perspective, liquid sprays represent a turbulent flowfield with large density gradients, with added complications due to the influence of surface tension. Moreover, measurements of spray flowfields are quite challenging, as optical diagnostics, which 
are favored for non-intrusive measurements of fluid flows, are often confounded by the strong interaction of visible light with phase boundaries [1]. Sprays for diesel injection are of particular interest. The spray structure is tied to pollutant formation [2], and these sprays feature small length scales, large velocity gradients, and small droplet sizes, which challenge experimental diagnostics and computational models. In applied diesel sprays, the action of vaporization will also alter the droplet size distribution.

While many measurements of the bulk behavior of diesel sprays have been performed, knowledge of the spray droplet size in the near-nozzle region is still relatively sparse. The nearnozzle region of diesel sprays (within roughly 50-100 nozzle diameters, depending on ambient density) is optically dense, which severely hampers optical diagnostics of these sprays. Some studies of diesel spray droplet size and velocity have been performed, using PDPA [3-5], light extinction [6,7], laser diffraction [8], scattering [9], and imaging [10,11], though generally in the spray far-field. Droplet size measurements close to the nozzle of a diesel spray are limited to a very thin region at the periphery of the plume where the optical density is modest, and the size distribution may be filtered by entrainment [11]. While these studies have provided valuable validation information for spray models, a better understanding of spray breakup requires droplet size measurements closer to the site of primary breakup. Under vaporizing and combusting conditions, complete evaporation of the fuel in a diesel spray can occur within 100 nozzle diameters of the injector [12]. Measurements of droplet size are needed upstream of this location to understand the influence of droplet breakup on the behavior of applied diesel sprays.

While recent work has used high-resolution LES methods to simulate diesel sprays [13], the computational expense of such methods is prohibitive for practical engine simulations; phenomenological methods predominate in these applications. While several phenomenological 
models have been proposed to calculate droplet size in diesel sprays, one of the most commonlyused models is based on the growth of Kelvin-Helmholtz and Rayleigh-Taylor waves on the liquid exiting the injector [14-16], and is commonly termed the KH-RT model. In this model, the primary spray breakup is hypothesized to occur from the formation of Kelvin-Helmholtz waves on the edges of the emitted fuel parcels, with a dispersion relation governing the size of the resulting droplets and the rate of breakup. Rayleigh-Taylor instabilities also act on the fuel, but only downstream of a "breakup length." While this model can be validated against droplet size data in the far-field, simply comparing modeled to measured droplet size in the far-field is insufficient to validate the physical breakup model; data in the near-field are needed, since this is the region in which primary breakup actually occurs. In applied sprays, droplet collisions also occur, which can lead to coalescence, which competes with breakup in determining droplet size.

X-ray diagnostics have been applied for the past 15 years to probe high-density sprays, taking advantage of the relatively weak interaction of x-rays with phase boundaries. While x-ray diagnostics have probed the density field of both quasi-steady state and transient sprays $[17,18]$ and visualized the morphology of certain sprays [19], quantitative measurements of spray droplet size are also desired by both the experimental and computational modeling communities. While $\mathrm{x}$-ray phase-contrast imaging can potentially be used for droplet sizing in dilute mixtures of large spray droplets, spatial resolution limits its use in dense fields of small $(<10 \mu \mathrm{m})$ droplets.

Small-angle $\mathrm{x}$-ray scattering (SAXS) is a well-established technique in the x-ray physics and material science communities for studying nanoscale particles [20-25]. The trend in scattering intensity vs. scattering vector $q$ can be used to quantitatively measure the size and shape of particles over a wide range of length scales. Its use for studying aerosols and sprays is less common. Lin et al. have used SAXS to study droplet size in a supercritical ethylene jet [26]. 
Wyslouzil, et al. have used SAXS to study aerosol formation in supersonic flows [27]. In both of these flows, droplets formed by homogeneous nucleation, and as such were much less than $1 \mu \mathrm{m}$ in diameter.

To the authors' knowledge, SAXS has not previously been used to study droplet size in a more conventional spray, where droplets are sheared from a bulk liquid structure into smaller droplets. The average droplet size in shear-driven sprays $(\geq 1 \mu \mathrm{m})$ is such that the scattering angles are quite small, making it difficult to separate the scattered $\mathrm{x}$-rays from the unperturbed $\mathrm{x}$ rays transmitted through the sample. Typical SAXS measurements are made by placing a twodimensional detector (such as an x-ray pixel array detector) a few meters downstream of the sample; the position of scattering on the detector is related to the scattering angle, and hence the scattering vector, by geometry. Ultra-small angle x-ray scattering (USAXS) is a specialized form of SAXS for measuring at low $q$. USAXS uses either a very long propagation distance for the scattering or uses diffraction from crystals as a filter for angle, rather than using propagation of the X-rays from sample to detector [28]. USAXS can measure an order of magnitude lower in scattering vector than SAXS [20,29]. This allows USAXS to measure far larger droplets than SAXS, making it a more attractive choice for measuring shear-driven sprays. However, this comes at the cost of greater experimental complexity and much slower measurements.

This study will describe measurements of Sauter mean diameter (SMD) in diesel sprays using USAXS. The authors believe these represent the first quantitative measurements of droplet size in the near-nozzle region of such optically dense sprays, and one of the first effective uses of x-ray diagnostics to measure the size of droplets created by shear-driven atomization. The theoretical basis of these measurements will be described, as well as the experimental setup. The results of the measurements and how SMD varies with respect to several important independent 
variables will also be detailed.

\section{SAXS Theory}

A brief overview of the relevant theory for SAXS, and by extension USAXS, will be given here, as the difference between the techniques lies in the detection system, not the scattering process itself; more detail can be found in other references [20,30]. SAXS is based on the principle of interference of scattered x-ray waves from electrons in materials. As X-rays are scattered by atoms in a material, constructive and destructive interference is seen between scattering from nearby atoms, depending on the angle of scattering and the relative positions of the atoms. This interference leads to an angular dependence of the scattering pattern, which for particles (solid particles or liquid droplets) is related to the particle shape and size.

The scattering pattern in SAXS is a function of a scattering vector $\boldsymbol{q}$, which is derived from the wavevectors of the incident and scattered x-rays, as depicted in Fig. 1. If one assumes that the scatterers are randomly oriented, the scattering is axisymmetric, and only the magnitude of $q$ matters. The scattering vector is related to the scattering angle and the x-ray wavelength by:

$$
q=\frac{4 \pi}{\lambda} \sin \theta
$$

where $\lambda$ is the $\mathrm{x}$-ray wavelength and $2 \theta$ is the angle between the incident and scattered photons. The typical units for $q$ are $\AA^{-1}$, as x-ray wavelengths are typically on the order of $1 \AA$. The value of $q$, or rather $1 / q$, also defines a length scale which SAXS probes. In order to derive a scattering signal, a density difference must exist on the same length scale as $1 / q$. 


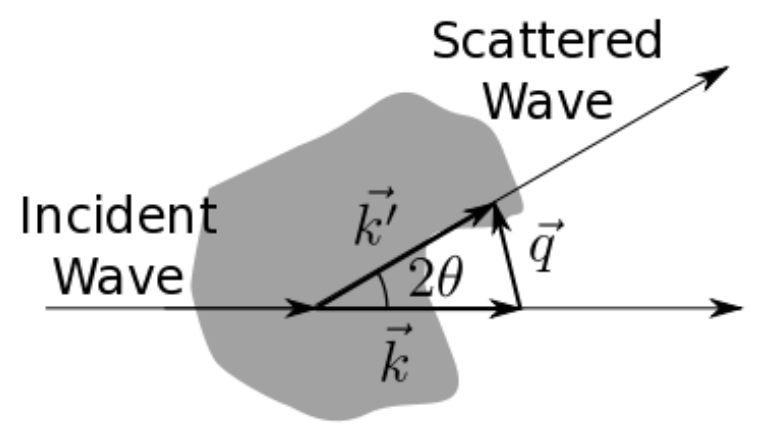

Figure 1: Derivation of scattering vector $q$ from the incident and scattered wavevectors

The intensity of scattered x-rays can be found by the following formula, assuming $100 \%$ detection efficiency $[20,30]$.

$$
I_{\text {scat }}(q)=I_{0} t \tau \Delta \Omega A_{\text {beam }} \frac{d \Sigma}{d \Omega}(q)
$$

In this equation, $t$ is the sample thickness, $\tau$ is the sample transmission, $\Delta \Omega$ is the solid angle of the detector, $A_{\text {beam }}$ is the area of the beam, and $d \Sigma / d \Omega$ is the differential cross-section per unit volume of sample. In an experiment, all quantities other than the differential cross-section can be measured, which allows the scattering data as a function of $q$ to be used to find the differential cross-section as a function of $q$.

The importance of the differential cross-section is that it can be related to the particle size and shape analytically. For a spray, we expect a wide range of droplet sizes. While fitting procedures can be used to determine particle size distributions [26], for the present analysis, two limiting cases will be used. In the limit of low $q$ (small scattering angles), Guinier's law applies:

$$
\frac{d \Sigma}{d \Omega}(q)=N V^{2} \Delta \rho^{2} e^{-\frac{q^{2} R_{G}{ }^{2}}{3}}, q R_{G}<1.5
$$

In this equation, $N$ is the number of particles per unit volume of the sample, $V$ is the particles' volume, $\Delta \rho$ is the scattering length contrast of the particles compared to the medium surrounding 
them, and $R_{G}$ is the radius of gyration of the particles. From quantitative measurements of this region, the particle (i.e., droplet) size and number density can be determined.

The second limiting case is for large $q$ (large scattering angles), termed Porod's law. At large $q$, the probed length scale is much smaller than the particle diameter. At these scales, SAXS probes the total surface area; it is only near the surface that one will see a density difference on the same length scale as $1 / q$. For spherical particles with sharp interfaces, the relationship between differential cross-section and scattering becomes:

$$
\frac{d \Sigma}{d \Omega}(q)=2 \pi \Delta \rho^{2} S q^{-4}, q R>10
$$

where $S$ is the total surface area per volume of sample. The particles need not be perfect spheres, so long as they are roughly similar sizes in all dimensions. The exponent of this power law can be used to understand the shape of the particles (spheres, rods, or flat plates), as well as whether the interface between the particles and the ambient environment is sharp or diffuse. Since it is the total surface area that is measured, if the total droplet volume in the sample can be determined (such as with x-ray radiography [18]), the SMD of the system can be determined trivially.

Figure 2 shows the theoretical scattering from two fields of small spherical droplets [20] of the size range expected for shear-driven atomization, both of which have the same liquid volume fraction. Note the logarithmic scale on both axes. The horizontal asymptote at low $q$ indicates that the scattering follows Guinier's law in this region, and the sloped asymptote at higher $q$ indicates scattering following Porod's law. As droplet diameter decreases, the Guinier region extends to higher $q$ linearly with droplet diameter, and the magnitude of scattering in the Porod region increases (due to the increase in droplet surface area/volume); smaller particle size leads 
to scattering at larger scattering angles. Ideally, if one could obtain a scattering curve over a wide enough range, such as ones shown in Fig. 2, one could use the shape of the curve alone to determine the particle size; quantitative measurements of scattering intensity would not even be needed. However, note that the Porod region begins at $q=10^{-4}-10^{-3} \AA^{-1}$. This value of $q$ is less than what can be reliably measured with a conventional SAXS instrument [20]. While Porod's law can be used by itself to determine the SMD of the spray, this requires quantitative measurements of the differential cross section, which are more challenging than qualitative measurements of scattering curve shape.

Both of these facts argue for the use of USAXS. USAXS can measure an order of magnitude lower in $q$ than a SAXS instrument, providing a greater opportunity to see Guinier behavior from small droplets, if such droplets exist. Moreover, USAXS by its nature provides quantitative measurements of scattering over several orders of magnitude.

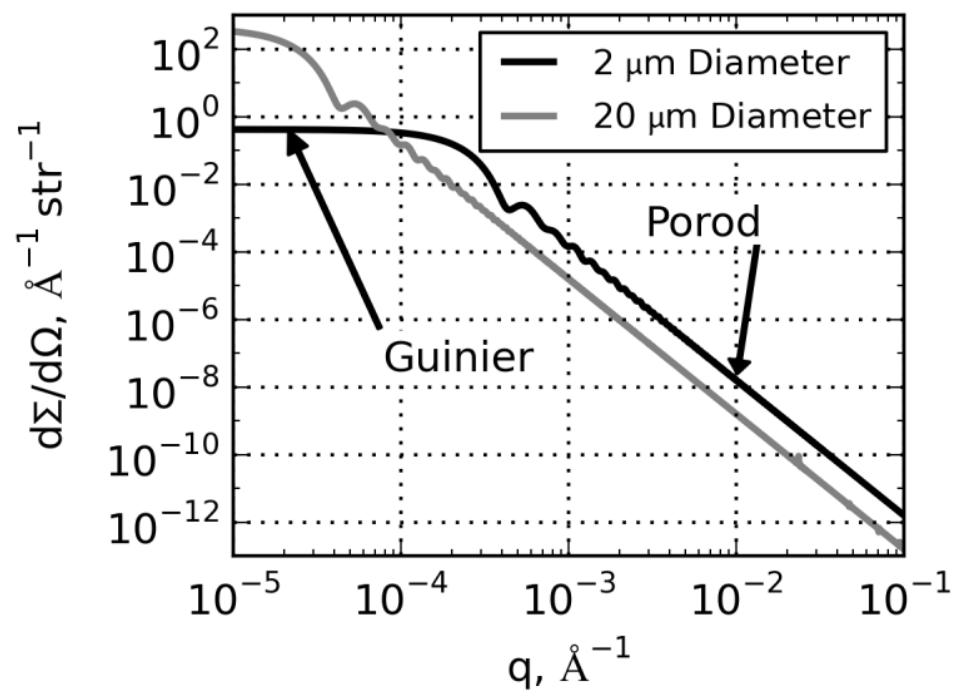

Figure 2. Simulation of scattering from a series of spheres of diameter from 1.6 to $2.4 \mu \mathrm{m}$ in diameter and from 16 to $24 \mu \mathrm{m}$ diameter. Assumed scattering length contrast matches that of the 
calibration fluid used in the current study. Droplet density $=1000$ droplets per $\mathrm{mm}^{3}$ for 2

micron droplets, 1 droplet per $\mathrm{mm}^{3}$ for 20 micron droplets.

\section{Materials and Methods}

The USAXS measurements were performed at the 9-ID and 15-ID beamlines of the Advanced Photon Source at Argonne National Laboratory, which were equipped with a BonseHart instrument [29], depicted schematically in Fig. 3. In this instrument, monochromatic x-rays diffract from a set of collimating crystals, which act to collimate the beam, severely restricting the divergence of the beam. A matching set of analyzer crystals are then scanned in angle to measure the scattering intensity as a function of angle, with the crystals acting as a narrow angular filter. X-rays at $17 \mathrm{keV}(15$-ID) and $17.9 \mathrm{keV}$ (9-ID) in a 100 x $500 \mu \mathrm{m}$ beam (V x H) were collimated with a pair of Si (220) crystals, passed through the spray, and were filtered with another pair of $\operatorname{Si}(220)$ analyzer crystals. Collection of data below $q \approx 1 \times 10^{-4} \AA^{-1}$ was not possible due to the finite angular resolution of the collimating and analyzer crystals, while low signal level precluded analysis for $q>1 \times 10^{-2} \AA^{-1}$. The upper limit of the $q$ range is not problematic, since no features are expected at higher $q$. The range of droplet sizes that can be probed based on the shape of the scattering curve alone will range from approximately $1 \mu \mathrm{m}$ to $10 \mathrm{~nm}$. This range is significantly extended when the quantitative scattering data are used, as discussed below. Data were taken in steps of $q$ of $\approx 1 \times 10^{-5} \AA^{-1}$ at low $q$, with wider spacing at higher $q$. The scattered intensity was converted to a quantitative measurement of differential cross-section for further analysis using the Irena data analysis package [31]. 


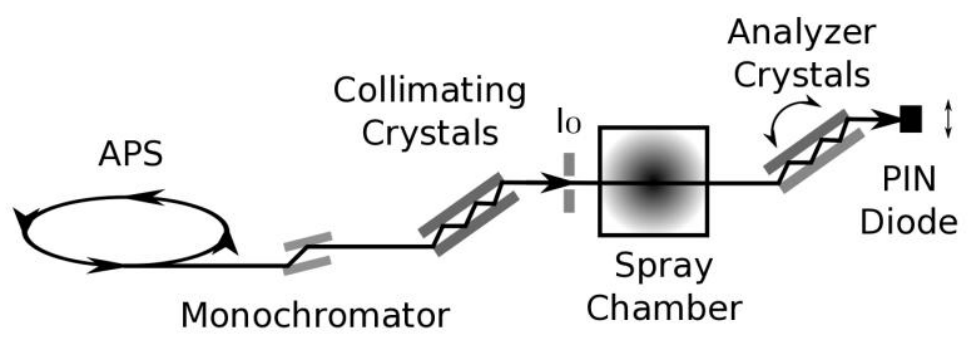

Figure 3: Schematic of USAXS experimental setup

To determine the spray density, time-resolved x-ray radiography of the sprays was performed at the 7-BM beamline of the APS at $8 \mathrm{keV}$ photon energy [32] under the same conditions as the USAXS measurements. The x-ray absorption was converted into a projected density (mass/area) of fuel in the path of the x-ray beam using the Beer-Lambert law $[18,33]$.

Given the novel application of USAXS to the study of shear-driven sprays, careful consideration must be given to calibration and error analysis. Calibration of the USAXS measurements is needed for both scattering angle and scattering intensity. The scattering angle is measured by physically rotating the analyzer crystals on a high-precision encoderinstrumented rotation platform. The USAXS instrument measures the scattering above and below the incident beam, providing both a direct measurement of the zero point for scattering angle and measuring the effectiveness of the angular filtering provided by the analyzer crystals, which defines the lowest $q$ that can effectively be measured. As such, the calibration of scattering angle is quite direct. In terms of scattering intensity, the instrument uses silicon photodiodes, previously demonstrated to be linear over several orders of magnitude of $\mathrm{x}$-ray flux [34], to directly measure the incident and scattered x-ray flux. From these values, scattering intensity values are calculated directly from first principles. Indeed, the USAXS instrument used in this study is used as an absolute calibration standard in the small angle x-ray scattering community [35]. Furthermore, several recent papers have demonstrated good quantitative 
agreement in particle sizing measurements between USAXS and several other particle sizing techniques, including electron microscopy, gas absorption, optical microscopy, and atomic force microscopy [20-22, 36].

There are three major sources of error in these measurements: noise in the scattering measurements, noise in the radiography measurements, and mismatch between the scattering and radiography measurements. The noise in the scattering measurements causes an uncertainty of approximately $1 \%$ in the specific surface area measurement, based on a linear fit to the scattering data. The precision of the radiography measurements is at worst $\pm 2 \%$ of the mean value, judged by examining the noise in the radiography measurements in regions outside the spray. To avoid mismatch in the positioning between scattering and radiography measurements, transverse scans at a fixed $q$ were performed; the scattering profile measured across the spray is analogous to the radiography signal, and as such the peak value in scattering provides a measure of the spray axis location. The authors estimate an approximately $\pm 5 \%$ uncertainty remaining in the SMD values due to possible positioning errors. Adding in quadrature, the resulting uncertainty of the SMD measurements is approximately $6 \%$.

The sprays were produced by a diesel common-rail injection system and standard solenoid-actuated diesel injectors firing at a $3 \mathrm{~Hz}$ repetition rate. The USAXS data acquisition was gated to accept data only during the steady-state portion of each injection event. As such, the duty cycle of data collection was quite low; each measurement location required approximately 1 hour of time to complete. Relatively long injection durations $(2.5-5 \mathrm{~ms})$ were used to allow for sufficient time in the steady-state period of the spray event to improve the duty cycle of the measurements. Background measurements were taken over a $50-80 \mathrm{~ms}$ time frame before injection events to eliminate signal from residual spray droplets from previous spray 
events, though the scattering curves for the background scans were virtually identical both to each other and to scans taken with clean gas in the chamber. The injector was fitted to a chamber with $0.5 \mathrm{~L}$ internal volume, pressurized with $\mathrm{N}_{2}$ at $25-28{ }^{\circ} \mathrm{C}$ with a purge flow of approximately 4 standard L/min. Due to the low chamber temperature, low vapor pressure of the fuel, slow purge rate, and repeated firing of the injector into the chamber gas, only minimal fuel vaporization is expected. Polyimide windows provided x-ray optical access.

Four axial single-hole nozzles of a similar design were used for this study, with $84,89,110$, and $180 \mu \mathrm{m}$ exit diameter, respectively. The two smallest nozzles were the 210675 and 210679 injectors of the Engine Combustion Network (ECN) Spray A effort [33,37]. Other than the diameter, all nozzles had a similar design: a nominally circular orifice approximately $1 \mathrm{~mm}$ in length, with hydroerosion applied to smooth the entrance region of the orifice, and with a design discharge coefficient of 0.86 . The nozzles are slightly converging to reduce the likelihood of cavitation in the nozzle, with a nominal reduction of nozzle diameter of $15 \mu \mathrm{m}$ between the orifice inlet and exit. In addition, a 3-hole diesel nozzle (injector 211201 of the ECN Spray B effort) with an orifice diameter of $94 \mu \mathrm{m}$ was also studied.

Experiments were conducted in several stages. Limited tests of the 110 and $180 \mu \mathrm{m}$ nozzles were conducted at the 15-ID beamline using a diesel calibration fluid as the injected liquid. Tests of the 210679 injector at 15-ID and the 210675 and $110 \mu \mathrm{m}$ nozzles at the 9-ID beamline were conducted using n-dodecane as the injected liquid. Fuel properties are listed in Table 1. Experiments were performed at seventeen combinations of nozzle, injection pressure $\left(\mathrm{P}_{\mathrm{L}}, 50\right.$ to $150 \mathrm{MPa})$, ambient pressure $\left(\mathrm{P}_{\mathrm{a}}, 0.5\right.$ to $\left.2.0 \mathrm{MPa}\right)$, and fuel type. For each set of conditions, measurements were performed from $x=1 \mathrm{~mm}$ downstream of the nozzle exit to the spray farfield ( $x \geq 20 \mathrm{~mm}$ ) along the spray axis. Limited measurements transverse to the spray axis have 
also been performed. It should be noted that fuel bypass through the injector led to heating of the injector, and hence the injected fuel, at higher injection pressures. The injector temperature ranged from $60-70^{\circ} \mathrm{C}$ at $150 \mathrm{MPa}$ injection pressure to $35-40^{\circ} \mathrm{C}$ at $50 \mathrm{MPa}$ injection pressure; given the relatively long test times and repeated injections of fuel through the injector, this should reasonably approximate the temperature of the injected fuel.

Table 1: Fuel properties at $25^{\circ} \mathrm{C}, 1 \mathrm{bar}$

\begin{tabular}{lcc} 
& Calibration Fluid & n-dodecane \\
\hline Density, $\mathrm{kg} / \mathrm{m}^{3}$ & 811 & 746 \\
Viscosity, $\mathrm{m}^{2} / \mathrm{s}$ & $3.2 \times 10-6$ & $1.8 \times 10-6$ \\
Surface Tension, $\mathrm{N} / \mathrm{m}$ & 0.0273 & 0.025
\end{tabular}

\section{Results}

Examples of the differential cross-section vs. scattering vector $q$ are shown as solid symbols in Fig. 4; these results are representative of all of the conditions tested, and can be directly compared to the theoretical scattering curves shown in Fig. 2 and in dotted lines on Fig. 4. Unlike the model curves, there is no horizontal asymptote in the experimental data at low $q$. The droplet size is large enough that despite the greater range in $q$ afforded by the use of USAXS, the Guinier plateau region of the scattering curve occurs at a smaller $q$ value than could be measured with the instrument. Instead, the scattering curves show only the Porod's law relationship between scattering and $q$. This implies that the droplet sizes must be greater than or equal to roughly $1 \mu \mathrm{m}$ (see Fig. 2), otherwise more of the Guinier region would be visible in the measurements. A model curve for an array of spherical scatterers of $1 \mu \mathrm{m}$ diameter is included in 
Fig. 4 for reference. The measured slopes of the scattering curves match the expected Porod's law exponent for isolated parcels of roughly equal size in all dimensions. This both provides information regarding the morphology of the liquid and confirms that the surface area can be calculated using Porod's law (Eq. 4).

At $\mathrm{P}_{\mathrm{L}}=150 \mathrm{MPa}$, some deviation from Porod's law is evident at the lowest $q$ values $(q<8 \mathrm{x}$ $10^{-4}$ ), suggesting some population of droplets with diameter $\sim 1 \mu \mathrm{m}$. Attempts to fit a combined Guinier-Porod curve to these data yield a Guinier radius of gyration of $1.5-3 \mu \mathrm{m}$, with a great deal of uncertainty on the Guinier fit parameters. Given that the sprays are expected to be highly polydisperse and only a small fraction of the Guinier curve has been measured, these Guinier fits are not expected to be usable for quantitative analysis. The presence of some deviation from Porod's law at low $q$ in the curve at higher injection pressure qualitatively suggests smaller droplet sizes are present than are seen at lower injection pressure, a trend which is probed quantitatively below.

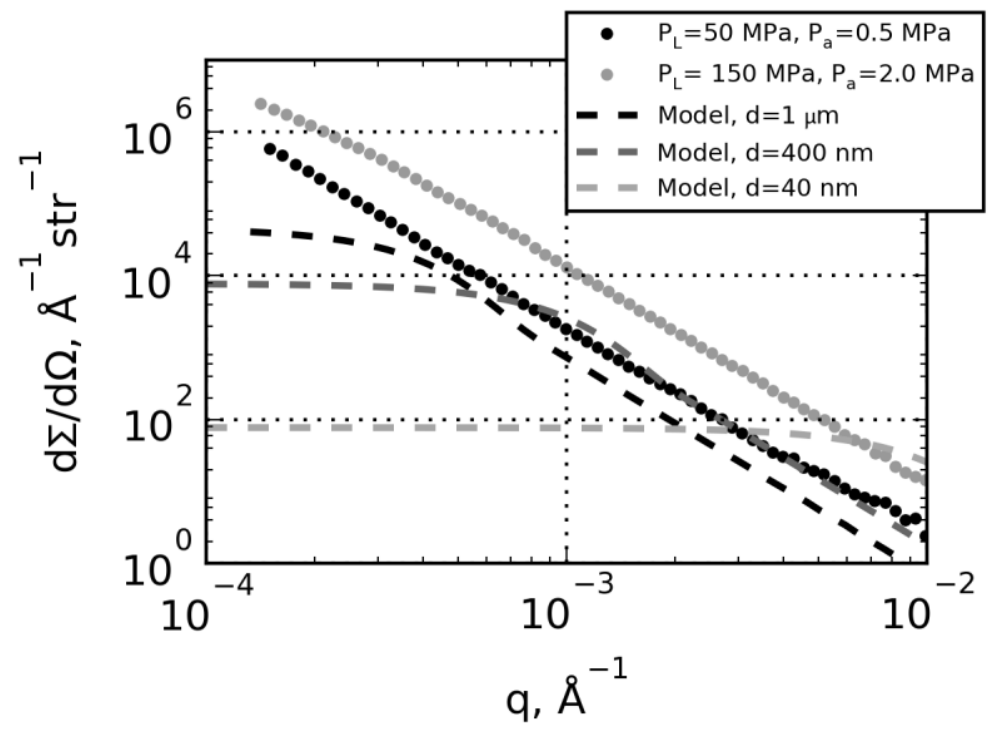

Figure 4: USAXS scattering curves for two spray cases for nozzle diameter $d=110 \mu \mathrm{m}, x=8$ mm with calibration fluid as the injected liquid. For comparison, KH-RT model curves for 
droplets with a Gaussian size distribution ( $\sigma=25 \%$ of mean diameter) and a volume fraction of $1 \times 10^{-3}$ are included. Both the data and model are slit-smeared.

The KH-RT model of spray breakup (as discussed in the Introduction) predicts the creation of droplets from aerodynamic waves that would be small enough to be easily seen in the scattering curves. One can compute a crude estimate of the child droplet size created immediately downstream of the nozzle exit by simply applying the KH-RT model equations [14], assuming inviscid flow through the injection nozzle to compute the liquid speed, using a parent droplet the same diameter as the nozzle, and using typical model constant values from the literature; note that these estimates are for comparison only, and are not the result of 2-D or 3-D CFD modeling. For the two conditions plotted in Fig. 4, the KH-RT model would predict droplet diameters of approximately 400 and $40 \mathrm{~nm}$ at $\mathrm{P}_{\mathrm{L}}=50 \mathrm{MPa}$ and $150 \mathrm{MPa}$, respectively. Modeled scattering curves for droplets of these sizes are shown in Fig. 4 as dashed lines. If a large population of nanoscale droplets existed, they would cause the measured scattering curves to visibly and significantly deviate from the Porod behavior seen experimentally (solid lines). As no such deviations are seen, the current data strongly suggest that there is no large population of nanoscale droplets in these sprays. Similar scattering curves are seen at all other positions measured; there is no position at which the scattering curve shape deviates significantly from Porod's law, except for the slight deviations at low $q$ noted above, which appear to be indicative of micron-scale droplets.

The large size of the spray droplets compared to the $q$ range probed by the USAXS instrument precludes using the shape of the scattering curve alone to determine the average droplet size, other than to demonstrate the absence of large populations of nanoscale droplets. To determine the droplet size quantitatively, Porod's law is applied to determine the specific 
surface area of the spray [31]. These measurements of surface area can be used to measure fields of larger droplets than a shape-based analysis alone, but do not give droplet size directly. To convert the specific surface area found by Porod's law to a droplet size, the liquid mass/area measured by x-ray radiography is used. Figure 5 shows an example of the measurements of spray surface area (in terms of surface area per unit area of the x-ray beam) and projected density (in terms of mass per unit beam area). These results are similar to those seen in all of the measurements: the surface area increases near the nozzle, reaches a maximum, then decreases, while the projected density decreases nearly monotonically. The overall surface area/liquid volume was converted to an SMD of the droplets.

A few limitations in these measurements must be noted. The Porod analysis used in this work does not assume that the droplets are spherical, but does assume that the droplets are randomly oriented, since scattering is only measured in one plane. At the closest positions to the nozzle exit, this may not be a valid assumption, since ligaments stripped from the liquid jet may be preferentially oriented. Farther downstream, the turbulent nature of the jet is expected to randomize the orientation of the droplets and ligaments. Second, the sensitivity of both the USAXS and radiography measurements are relatively poor in dilute regions of the spray; for this reason, the current measurements focus on the near-nozzle region near the spray axis. Third, as both the scattering and radiography measurements are pathlength-integrated, the SMD measurements are pathlength-integrated, and as such probe the droplet size on the entire beam path through the spray. Finally, this measurement provides the SMD of the spray, not a droplet size distribution, like would be given by some optical droplet sizing techniques. Depending on the droplet size distribution, the SMD may or may not be representative of the most dynamically important droplets at any given position in the spray. 


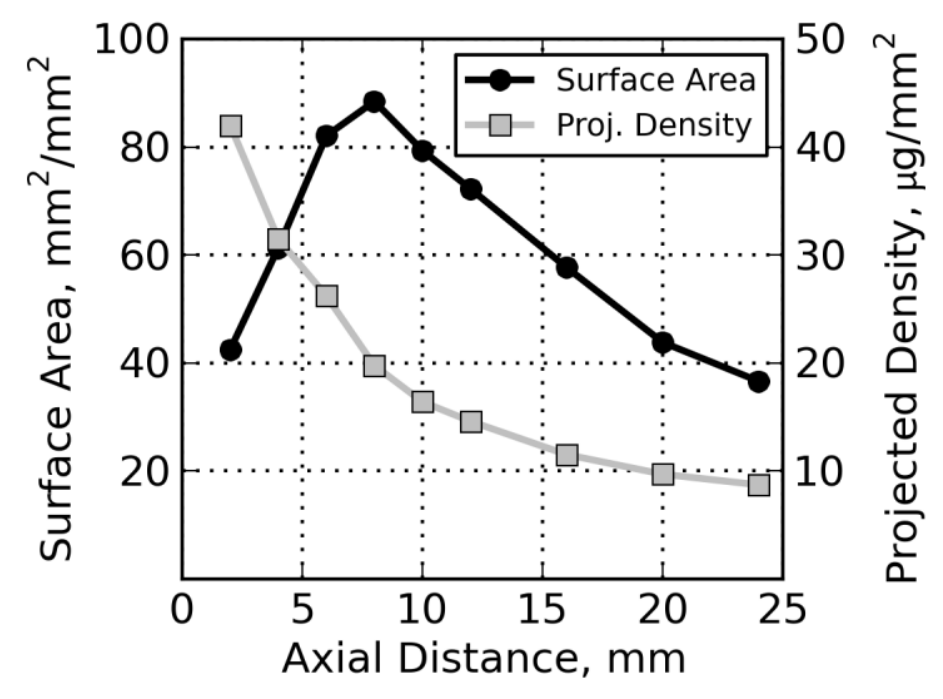

Figure 5: Trends in measured surface area and projected density along the spray axis, $110 \mu \mathrm{m}$ diameter nozzle, $P_{L}=130 \mathrm{MPa}, P_{a}=1.2 \mathrm{MPa}, n$-dodecane fuel

To demonstrate the repeatability of the USAXS measurements, Fig. 6 shows the SMD results along the spray axis for the two ECN Spray A injectors and one Spray B injector tested, all performed at $\mathrm{P}_{\mathrm{L}}=150 \mathrm{MPa}, \mathrm{P}_{\mathrm{a}}=2.0 \mathrm{MPa}$ with $\mathrm{n}$-dodecane fuel. These injectors were designed to have identical nozzle diameters, though the actual nozzle diameters are slightly different [37]. Though the two measurements were conducted during different measurement campaigns and use different injectors, the SMD results for the two Spray A injectors are remarkably similar. The Spray B injector (211201) shows similar trends in droplet size, but a more rapid decrease in SMD near the nozzle, perhaps due to the more complex inflow condition for the orifice in a multihole nozzle compared to the single-hole Spray A injectors.

These data also show many features present in all of the USAXS SMD data. The SMD rapidly declines near the nozzle. Even at axial distance $x=1 \mathrm{~mm}$ downstream of the nozzle, the SMD is already $<10 \mu \mathrm{m}$. It should be noted that given the high liquid volume fraction so near the nozzle, the fuel is not expected to be dispersed as isolated droplets [38], so the SMD must be 
interpreted carefully. Rather than giving a size of individual droplets, it is an indication of the surface area to volume ratio in the spray. At first glance, such a small SMD near the nozzle seems unlikely, given that the liquid jet has had little space to aerodynamically interact with the ambient gas. However, visible light shadowgraphs of the 210675 injector [39] show only a small region of clear liquid with a relatively smooth interface in the jet immediately outside the nozzle exit. Surface irregularities, which manifest themselves as opaque regions of the spray, are present within even a single nozzle diameter of the injector exit. Other recent visible light images of diesel sprays $[13,40,41]$ show similar results. The rapid initiation of surface irregularities will rapidly increase the liquid-gas surface area even in close proximity to the nozzle, leading to a rapid decrease in the SMD measured with USAXS.

After the rapid decline in SMD near the nozzle, the droplet size becomes much more stable as $x$ increases further. In many cases, the SMD reaches a minimum value, with a subtle increase in SMD as $x$ increases further. It should be noted that this increase in droplet size is quite weak, especially when compared to the scatter in the experimental data. 


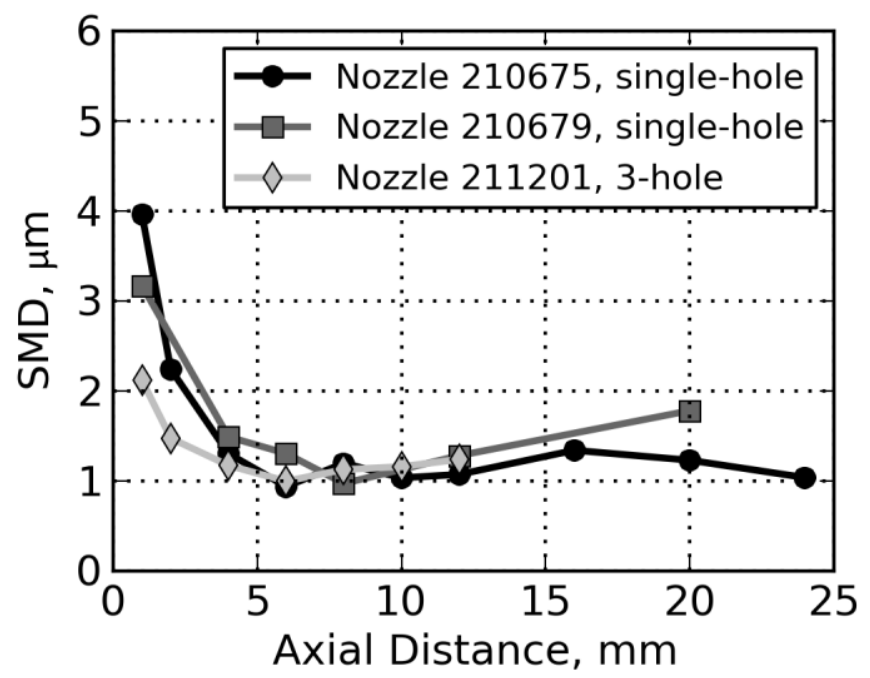

Figure 6: USAXS spray SMD measurements for the ECN Spray A and Spray B injectors on the spray axis, $P_{L}=150 \mathrm{MPa}, P_{a}=2.0 \mathrm{MPa}$, injecting $n$-dodecane.

It is useful to compare the droplet sizes seen in these measurements with those from other studies. Optical far-field measurements of droplet size give similar droplet sizes to those seen in this study $[9,42]$. Moreover, recent LES simulations of diesel injection at similar injection pressures [13] have shown the formation of large populations of $\sim 2$ micron droplets starting within 1 nozzle diameter of the injector exit, consistent with the results of this study.

Figure 7 shows the droplet SMD vs. $x$ at different ambient pressures for two of the nozzles, both using n-dodecane fuel at $150 \mathrm{MPa}$ injection pressure. It is clear from the plots that, while the ultimate far-field droplet size is similar for different ambient densities, the axial distance at which this droplet size is reached decreases strongly as ambient density increases. This is logical: one expects spray breakup to rely on either shear or deceleration of the liquid to promote breakup, both of which are enhanced by higher ambient density. Measurements of the $180 \mu \mathrm{m}$ nozzle with calibration fluid show similar trends with ambient density. 


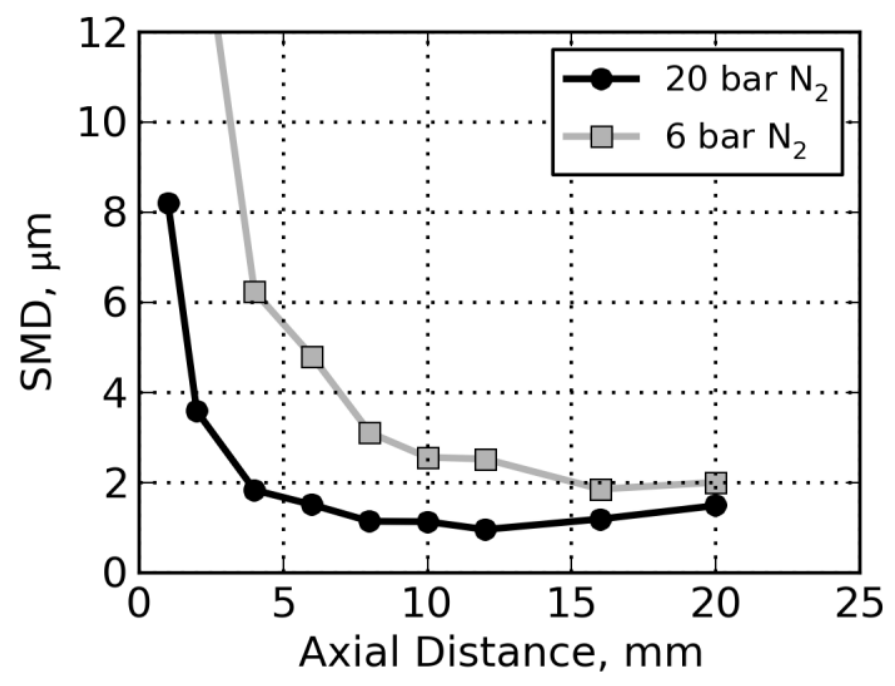

a)

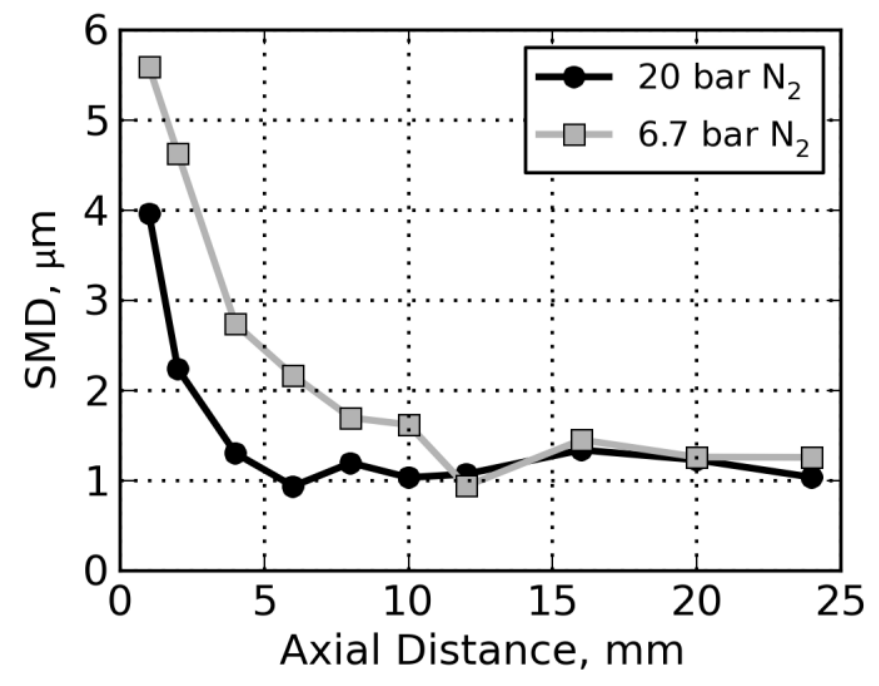

b)

Figure 7: Spray SMD vs. $x$ along the spray axis with $n$-dodecane injected liquid at different $P_{a}$ for a) $110 \mu \mathrm{m}$ diameter nozzle and b) ECN injector 210675 (single-hole, 89 um diameter) The injection pressure is expected to have a major impact on the droplet size; higher 
injection pressure increases the shear between the ambient gas and the liquid, as well as the deceleration on the liquid. The influence of injection pressure for the 210675 Spray A injector with n-dodecane fuel at 2.0 MPa chamber pressure is shown in Fig. 8. Increased injection pressure decreases the droplet size, especially in the regions nearest the nozzle exit. The increase in droplet size after this minimum is also much more pronounced at lower injection pressure. The location at which the minimum droplet size occurs, however, changes little with injection pressure. Similar trends are seen for the $110 \mu \mathrm{m}$ diameter nozzle, both with n-dodecane and calibration fluid as the injected fluids.

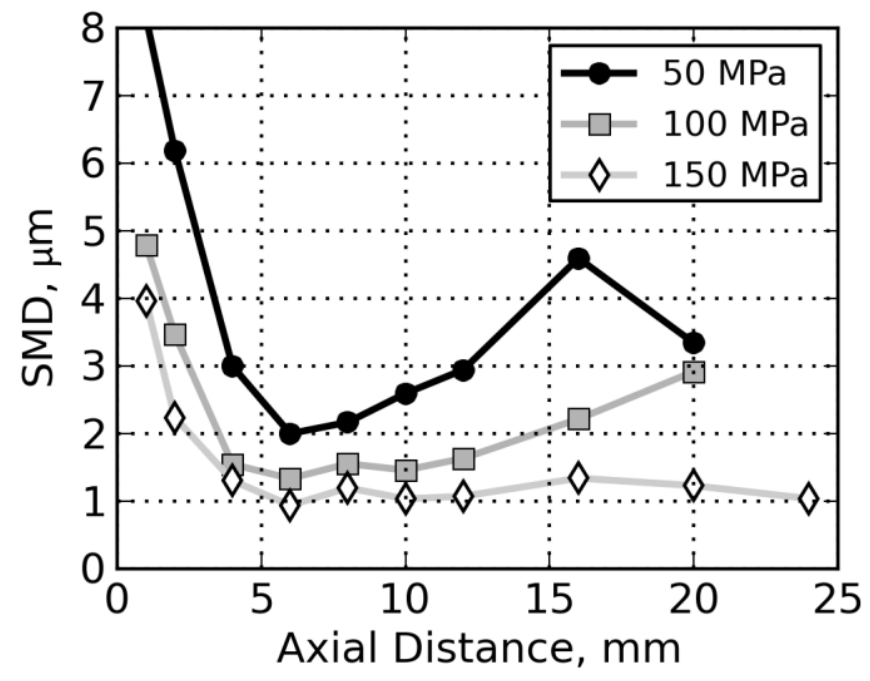

Figure 8: Spray SMD along the spray axis vs. $x$ for the ECN 210675 injector (single-hole, $89 \mu \mathrm{m}$ diameter) at various $P_{L}, P_{a}=2.0 \mathrm{MPa}$, injecting $n$-dodecane

In addition to the axial trends in droplet size, the presence of radial gradients in droplet size is also of interest. While the current pathlength-integrated measurements are not well-suited to measure such gradients, limited measurements at several transverse positions have been undertaken for the ECN 210675 injector at $150 \mathrm{MPa}$ injection pressure, 2.0 MPa ambient 
pressure. The results are shown in Fig. 9. The measured points range from the spray axis (left hand end of each curve) to relatively dilute regions of the spray. There is significant scatter between points, with no clear trend in droplet size with transverse position in the spray, though there may on average be an increase in droplet size with transverse distance. The current data do not support the presence of strong gradients in droplet size within the dense regions of the spray. On the other hand, due to the limited ability of these measurements to probe highly dilute regions of the spray, it is possible that SMD gradients exist between the spray core and the optically thin spray periphery, which is more typically measured with optical diagnostics [10].

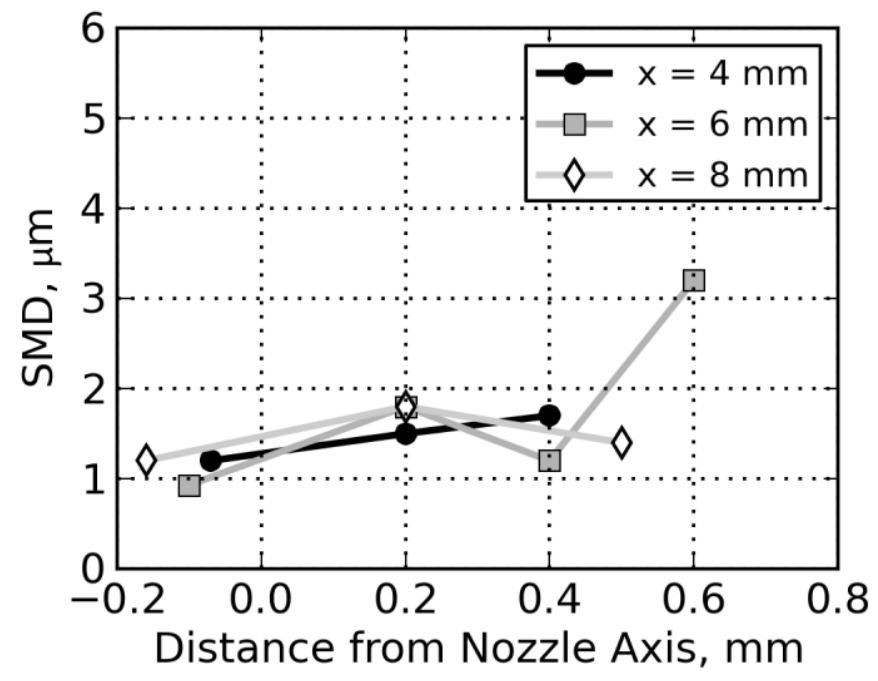

Figure 9: Spray SMD vs. distance from the nozzle axis for the ECN 210675 injector (single-hole, $89 \mu \mathrm{m}$ diameter), $P_{L}=150 \mathrm{MPa}, P_{a}=2.0 \mathrm{MPa}$, injecting $n$-dodecane

Upon examining these data, a clear question is why the droplet size plateaus at a minimum in the range $0.5-2 \mu \mathrm{m}$. To better understand these data, it is useful to rescale the droplet data to a gas phase Weber number $\mathrm{We}_{\mathrm{g}}=\rho_{\mathrm{a}} \mathrm{V}^{2} d / \sigma$. Recently, $\mathrm{x}$-ray radiography has been used to determine an average axial velocity for a given $x$ location in steady-state sprays [18]. Using this velocity value and the measured SMD, an effective $\mathrm{We}_{\mathrm{g}}$ can be computed. The local relative 
velocity between the droplets and the gas is likely smaller than this average liquid velocity; unfortunately, a proper calculation of $\mathrm{We}_{\mathrm{g}}$ would require independent measurements of gas and liquid phase velocity, which are unavailable, especially in the optically dense core of the spray. While the $\mathrm{We}_{\mathrm{g}}$ calculated here is likely an overestimate of the true Weg, it at least provides a reasonable estimate of this value, and especially of the trends in $\mathrm{We}_{\mathrm{g}}$ with axial distance.

Two competing mechanisms are expected in these sprays: breakup and coalescence. Previous work has shown that droplets are stable against further shear-driven breakup when the gas phase Weber number $\mathrm{We}_{\mathrm{g}}=\rho_{\mathrm{a}} \mathrm{V}^{2} d / \sigma<12$ [43]. The trends in droplet coalescence are more complex [44], and depend on the relative liquid phase Weber number between the droplets and an impact parameter that accounts for the angle of impact between the droplets. At high relative We, the droplets seldom coalesce, instead rebounding from each other and often creating new satellite droplets. As the $\mathrm{We}_{\mathrm{L}}$ decreases, coalescence of droplets becomes more likely.

Figure 10 shows the droplet SMD and computed We for the $210675 \mathrm{ECN}$ injector at three different conditions. At $x=1 \mathrm{~mm}$, the droplet $\mathrm{We}_{\mathrm{g}}$ is quite high, though it must be borne in mind that the SMD is likely an average between small dispersed droplets and larger intact liquid structures at this location, so the $\mathrm{We}_{\mathrm{g}}$ of the large and small droplets will be quite different. By examining the two plots, it is clear that the minimum droplet size is reached when $\mathrm{We}_{\mathrm{g}}=80-200$. While these values are somewhat higher than the stable droplet size listed above, given that the relative velocity is likely overpredicted, the agreement is reasonable. These data support the view that the plateau in droplet size occurs because the droplets have simply become small enough for surface tension to resist further breakup. These $\mathrm{We}_{\mathrm{g}}$ values imply $\mathrm{We}_{\mathrm{L}}$ values far higher than the critical $\mathrm{We}_{\mathrm{L}}$ where significant droplet coalescence is expected, which may explain the relatively slow evolution of droplet size once a stable size is reached. 
Examination of the droplet $\mathrm{We}_{\mathrm{g}}$ also helps to explain the effects of changes in injection pressure and ambient density. For all $x$, lower injection pressure leads to lower $\mathrm{We}_{\mathrm{g}}$ values, allowing a stable $\mathrm{We}_{\mathrm{g}}$ to be reached at a higher droplet diameter, which helps to explain the larger ultimate droplet size at lower injection pressure. The lower We values at lower injection pressure may also explain why the droplet diameter rises more significantly in the spray far-field as the injection pressure drops: the $\mathrm{We}_{\mathrm{L}}$ values are lower at lower injection pressure, and hence closer to the critical $\mathrm{We}_{\mathrm{L}}$ where effective coalescence occurs. At lower ambient density, $\mathrm{We}_{\mathrm{g}}$ decreases more slowly as $x$ increases due to the weaker deceleration of the spray. The point where a stable $\mathrm{We}_{\mathrm{g}}$ is reached is farther downstream at lower ambient density, explaining the delayed evolution of the SMD as ambient pressure is reduced. Similar trends are exhibited for the $110 \mu \mathrm{m}$ nozzle. 


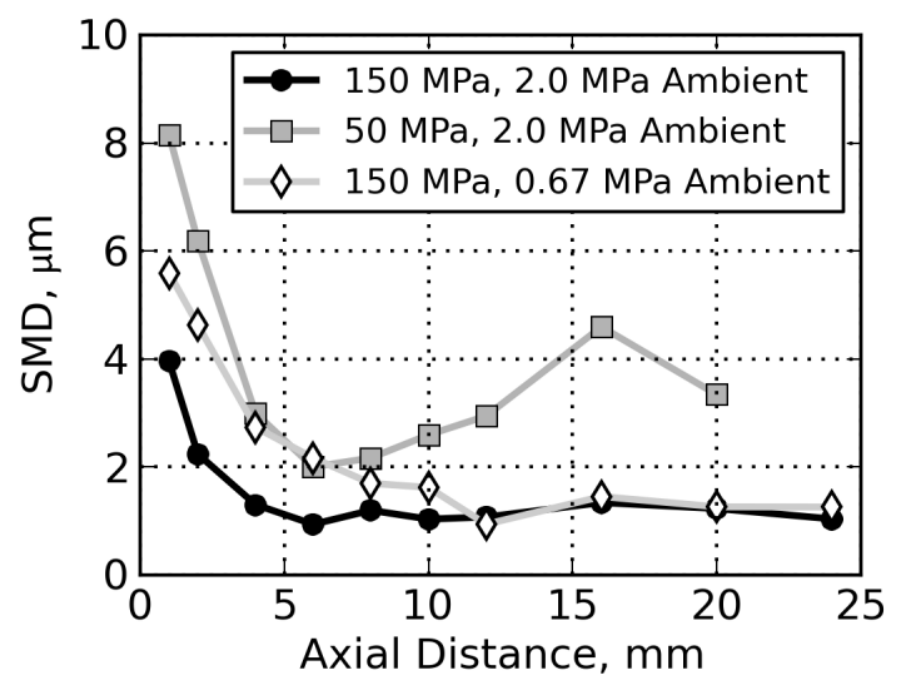

a)

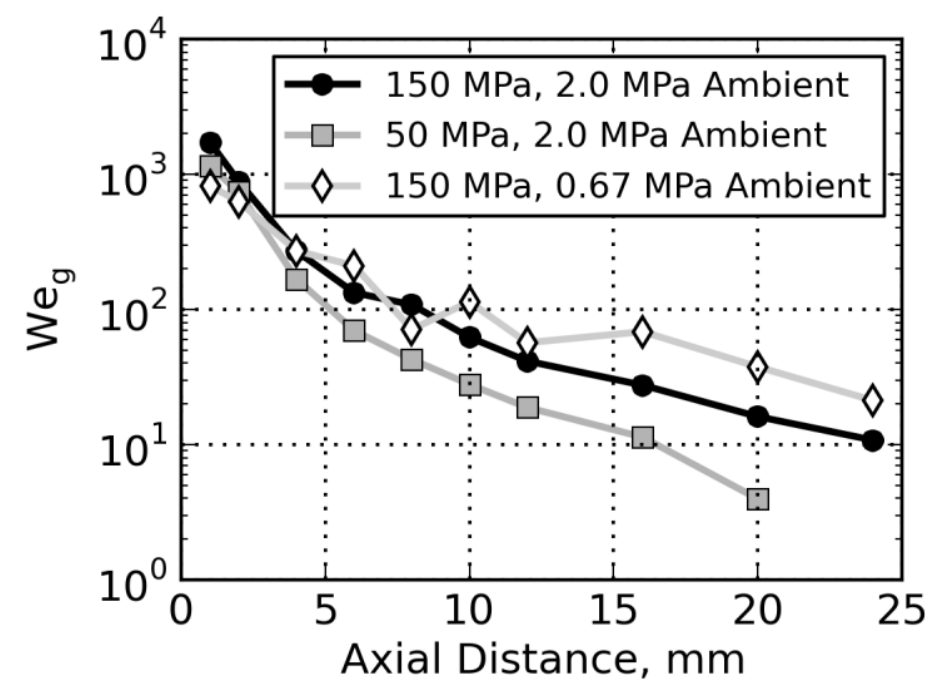

b)

Figure 10: a) SMD vs. $x$ and b) We vs. $x$ for the ECN 210675 nozzle (single-hole, $89 \mu \mathrm{m}$ diameter) along the spray axis with n-dodecane as the injected liquid 
Further evidence that $\mathrm{We}_{\mathrm{g}}$ is likely the controlling parameter of minimum droplet size come from examining the influence of the injected fluid. The minimum droplet size for calibration fluid for the $110 \mu \mathrm{m}$ diameter nozzle at $\mathrm{P}_{\mathrm{L}}=150 \mathrm{MPa}$ is somewhat smaller than that of $\mathrm{n}$ dodecane (1.1 vs. $1.9 \mu \mathrm{m}, \mathrm{P}_{\mathrm{a}}=0.5$ vs. $0.6 \mathrm{MPa}$, respectively), despite the fact that calibration fluid has a significantly higher viscosity. The KH-RT model predicts a significantly larger droplet size with calibration fluid than for n-dodecane due to calibration fluid's higher viscosity. The We of the droplets, however, is only weakly affected by the choice of liquid, since the density and surface tension of the two fuels are not markedly different. The fact that the minimum droplet size does not increase greatly with fluid viscosity suggests that this droplet size is not strongly tied to the KH-RT wave breakup mechanism.

To better quantify the relationship between the SMD and the pertinent independent variables (injection pressure, nozzle diameter, and ambient density), a power law fit was performed to the minimum droplet size found at each condition for all of the single-hole nozzle conditions where a clear minimum had been reached (i.e., all but one single-hole nozzle condition). To remove the influence of fuel properties, separate fits were performed for the n-dodecane and calibration fluid experiments; more data are needed to probe the influence of different fuel properties. For the calibration fluid, the fit equation across six conditions is:

$$
S M D_{\min } \propto P_{L}^{-0.66} d^{1.12} \rho_{a}^{-0.09}
$$

where $\rho_{\mathrm{a}}$ is the ambient density and $d$ is the nozzle diameter. The fit covariance of the power law exponents is less than $1 \%$ of the fitted value for the exponents of $\mathrm{P}_{\mathrm{L}}$ and $d$, indicating relatively high confidence in the exponent values. For the n-dodecane experiments, the fit equation across 8 conditions is: 


$$
S M D_{\min } \propto P_{L}^{-0.66} d^{1.22} \rho_{a}^{-0.25}
$$

The coefficients agree quite well for the two fuels, especially for injection pressure and nozzle diameter. These fits confirm the impressions seen in the plots: higher injection pressure and smaller nozzle diameter lead to smaller spray droplets, with ambient density having a smaller impact.

Given that ambient density is directly proportional to $\mathrm{We}_{\mathrm{g}}$, it is surprising that it has little impact in the fits for minimum droplet size. Recent $\mathrm{x}$-ray radiography measurements of similar

sprays have shown that trends in axial velocity vs. $x$ are best understood by normalizing $x$ by $\rho_{\mathrm{a}}{ }^{1 / 2}$ [18]. While increased $\rho_{\mathrm{a}}$ would initially appear to decrease the stable droplet size, it will also cause a more rapid decay of axial velocity; lower downstream velocity will lead to a lower We for a droplet of a given diameter, which should partially offset the effect of ambient density on $\mathrm{We}_{\mathrm{g}}$.

\section{Discussion}

From the above results, a conceptual model of the atomization process can be formed. Immediately upon exiting the nozzle, features with a characteristic size of a few $\mu \mathrm{m}$ are formed, though most of the liquid has not yet undergone breakup, so the measured SMD is still much larger than $1 \mu \mathrm{m}$. As the spray proceeds downstream, further breakup occurs, though as the spray decelerates through interaction with the ambient gas, the relative velocity between the liquid and gas decreases, reducing the droplet $\mathrm{We}_{\mathrm{g}}$ and causing breakup to slow down. Droplet coalescence competes with breakup, but quite inefficiently due to the high We, so breakup dominates over coalescence. Eventually, the spray decelerates enough that the droplets are relatively stable, causing a plateau in the SMD due to the low rate of additional breakup. As the 
spray further decelerates, coalescence becomes more efficient due to the lower We, causing a slow rise in SMD.

The absence of nanoscale droplets is a surprising finding from this study in light of the droplet sizes predicted by the KH-RT spray breakup model. Both the shape of the scattering curves and the quantitative SMD measurements suggest relatively few droplets smaller than the $0.5-2 \mu \mathrm{m}$ range; this contrasts sharply with the KH-RT model, especially near the nozzle. The production of a large population of nanoscale droplets would have profound implications on spray development, as their transfer of mass and momentum to the surrounding gas will be far more efficient than such transfer from larger droplets. Moreover, while the KH-RT model uses a breakup length at which Rayleigh-Taylor breakup is assumed to occur, there is no sign of any discontinuity in the SMD distribution with $x$. Indeed, the most rapid breakup is consistently found near the nozzle. It is clear that the KH-RT model does not qualitatively or quantitatively capture the primary breakup process. While the discussion in this paper has focused on comparison to the KH-RT model, other spray breakup models exist using both Lagrangian and Eulerian formulations. Other models may better agree with the droplet sizing results shown in this work.

It may be argued that nanoscale droplets, though not seen in the current measurements, may be created, but simply evaporate too quickly to be seen in the measurements. The current measurements cannot rule out this possibility. The current measurement conditions, however, with injection occurring repetitively into room-temperature gas with relatively slow purging, are far less favorable to evaporation than the evaporative spray conditions found in diesel engines. If such features evaporate under the current experimental conditions, it raises questions regarding their lifetime and dynamic importance in applied diesel sprays. It is also possible that nanoscale 
droplets are formed under different operating conditions than those probed, though the current conditions have used values of nozzle diameter, ambient density, nozzle shape, and fuel that are reasonably similar to those seen in applied diesel combustion.

Unlike existing optical measurements, the current measurements show the evolution of the droplet size in the near-nozzle region, including both the core and periphery of the spray. It is clear from these measurements that virtually the entire spray breakup process occurs in the region $x<12 \mathrm{~mm}$. The ability to measure droplet size in this region is particularly relevant in diesel sprays given the rapid vaporization and combustion of the fuel in diesel engines, as well as the link between spray structure and pollutant formation. For example, recent measurements have shown that the injected liquid is completely vaporized in the near-nozzle region under realistic operating conditions in diesel engines [12]. The current data provide a quantitative method to directly validate computational models of spray breakup, which have heretofore relied only on measurements of droplet size in the far-field region. That said, it is encouraging that the far-field droplet sizes found in this work are similar to those found in other recent measurements of diesel sprays using optical diagnostics [9,42].

\section{Conclusion}

The current study has demonstrated unique measurements of droplet SMD in shear-driven atomization using USAXS. Both quantitative and qualitative examination of the data show no support for the formation of nanoscale droplets. This is in contrast to a widely-applied spray model for diesel sprays. The droplet SMD rapidly decreases from a large fraction of the nozzle size to only a few microns in diameter as $x$ increases, then reaches a nearly steady-state with further increases in $x$. Increases in ambient pressure serve to accelerate the breakup of the spray, 
but do not strongly influence the final droplet size. Higher injection pressure does not strongly influence the location where the SMD plateaus, but does lead to smaller droplets. An empirical correlation to the data confirms the strong influence of injection pressure and nozzle diameter, as well as the weak influence of ambient density.

\section{Acknowledgements}

X-ray spray research at Argonne is supported by the DOE Advanced Combustion Program. We acknowledge team leaders Gurpreet Singh and Leo Breton for their support. These experiments were performed at the 7-BM, 9-ID, and 15-ID beamlines of the Advanced Photon Source, Argonne National Laboratory. ChemMatCARS Sector 15 is principally supported by the Divisions of Chemistry (CHE) and Materials Research (DMR), National Science Foundation, under grant number NSF/CHE-1346572. Use of the Advanced Photon Source, an Office of Science User Facility operated for the U.S. Department of Energy (DOE) Office of Science by Argonne National Laboratory, was supported by the U.S. DOE under Contract No. DE-AC0206CH11357. R. Payri was funded by a Fulbright visiting scholar grant in collaboration with the Ministry of Education, Culture and Sports of Spain (reference PRX14/00331) while performing this work. J.P. Viera was funded by the Spanish MINECO grant EEBB-I-15-0976 under project TRA2012-36932. The authors thank Lyle Pickett of Sandia National Laboratories for the use of several of the injector nozzles tested in this work.

\section{References}

[1] Smallwood G, Gülder Ö. Views on the structure of transient diesel sprays. Atom Sprays 2000;10:355-86.

[2] Flynn P, Durrett R, Hunter G, zur Loye AO, Akiyemi OC, Dec JE, Westbrook CK. Diesel combustion: an integrated view combining laser diagnostics, chemical kinetics, and empirical validation. SAE paper 1999-01-0509, 
1999.

[3] Koo JY, Martin J. Droplet sizes and velocities in a transient diesel fuel spray. SAE paper 900397, 1990.

[4] Yoda T, Tsuda T. Influence of injection nozzle improvement on DI diesel engine. SAE paper 970356, 1997.

[5] Payri R, Tormos B, Salvador J, Araneo L. Spray droplet velocity characterization for convergent nozzles with three different diameters. Fuel 2008;87:3176-82.

[6] Dan T, Takagishi S, Senda J, Fujimoto H. Effect of ambient gas properties for characteristics of non-reacting diesel fuel spray. SAE Paper 970352, 1997.

[7] Chang C, Farrell P. A study of the effects of fuel viscosity and nozzle geometry on high injection pressure diesel spray characteristics. SAE Paper 970353, 1997.

[8] Farrar-Khan JR, Andrews GE, Williams PT, Influence of nozzle sac volume on diesel spray droplet sizes. Proc Instn Mech Engrs 1992;206:239-48.

[9] Labs J, Parker T. Two-dimensional droplet size and volume fraction distributions from the near-injector region of high-pressure diesel sprays. Atom Sprays 2006;16:843-55.

[10] Heimgärtner C, Leipertz A. Investigation of the primary spray breakup close to the nozzle of a common-rail high pressure diesel injection system. SAE paper 2000-01-1799, 2000.

[11] Crua C, De Sercey G, Heikal M, Gold M. Dropsizing of near-nozzle diesel and RME sprays by microscopic imaging. ICLASS 12th Triennial International Conference on Liquid Atomization and Spray Systems, Heidelberg, Germany, 2012.

[12] Pickett LM, Genzale C, Bruneaux G, Malbec LM, Hermant L, Christiansen C, Schramm J. Comparison of diesel spray combustion in different high-temperature, high-pressure facilities. SAE Int J Engines 2010;3:156-81.

[13] Ghiji M, Goldsworthy L, Brandner PA, Garniya V, Hield P. Analysis of diesel spray dynamics using a compressible Eulerian/VOR/LES model and microscopic shadowgraphy. Fuel 2017:188:352-66.

[14] Beale JC, Reitz R. Modeling spray atomization with the Kelvin-Helmholtz/Rayleigh-Taylor hybrid model. Atom Spray 1999;9:623-50. 
[15] Lee C-S, Park, SW An experimental and numerical study on fuel atomization characteristics of high-pressure diesel injection sprays. Fuel 2002:81:2417-2423.

[16] Bravo L, Wijeyakulasuriya S, Pomraning E, Senecal PK, Kweon C-B. Large eddy simulation of high Reynolds number nonreacting and reacting JP-8 sprays in a constant pressure flow vessel with a detailed chemistry approach. J Energ Resour-ASME 2016:138:032207

[17] Eberhart CJ, Lineberry D, Fredrick Jr R, Kastengren AL. Mechanistic assessment of swirl coaxial injection by quantitative x-ray radiography. J Propul Power 2014;30:1070-9.

[18] Kastengren AL, Powell CF, Wang YJ, Im KS, Wang J. X-ray radiography measurements of diesel spray structure at engine-like ambient density. Atom Sprays 2009;19:1031-44.

[19] Moon S, Gao Y, Wang J, Fezzaa K, Tsujimura T. Near-field dynamics of high-speed diesel sprays: effects of orifice inlet geometry and injection pressure. Fuel 2014;133:299-309.

[20] Rieker T, Hanprasopwattana A, Datye A, Hubbard P. Particle size distribution inferred from small-angle x-ray scattering and transmission electron microscopy. Langmuir 1999:15:638-41.

[21] Kammler H, Beaucage G, Mueller R, Pratsinis S. Structure of flame-made silica nanoparticles by ultra-smallangle x-ray scattering. Langmuir 2004:20:1915-21.

[22] Brandt R, Petricevic R, Pröbstle H, Fricke J. Acetic acid catalyzed carbon aerogels. J Porous Mat 2003:10:171-8.

[23] Narayanan, T. Synchrotron small-angle x-ray scattering. In: Borsali R, Pecora R, editors. Soft Matter: Scattering, Imaging, and Manipulation. Dordrecht:Springer;2007.

[24] Hessler J, Seifert S, Winans R, Fletcher T. Small-angle x-ray studies of soot inception and growth. Farad Discuss 2001;119:395-407.

[25] Ilavsky, J., F. Zhang, A. J. Allen, L. E. Levine, P. R. Jemian and G. G. Long (2013). "Ultra-Small-Angle X-ray Scattering Instrument at the Advanced Photon Source: History, Recent Development, and Current Status." Metallurgical and Materials Transactions a-Physical Metallurgy and Materials Science 44A(1): 68-76.

[26] Lin KC, Ryan M, Carter C, Sandy A, Narayanan S, Ivasky J, Wang J. Investigation of droplet properties of 
supercritical ethylene jets using small Angle x-ray scattering (SAXS) technique,” 21st ILASS-Americas Conference, 2008 .

[27] Wyslouzil B, Wilemski G, Strey R, Seifert S, Winans RE. Small angle x-ray scattering measurements probe water nanodroplet evolution under highly non-equilibrium conditions. Phys Chem Chem Phys 2007;9:5353-58.

[28] Bonse U, Hart M. Tailless x-ray single-crystal reflection curves obtained by multiple reflection. Appl Phys Lett 1965:7:238-40.

[29] Ilavsky J, Jemian P, Allen A, Zhang F, Levine L, Long G. Ultra-small-angle x-ray scattering at the Advanced Photon Source. J Appl Cryst 2009;42:469-79.

[30] Glatter O, Kratky O. Small-angle x-ray scattering, London: Academic Press; 1982.

[31] Ilavsky J, Jemian P. Irena: tool suite for modeling and analysis of small-angle scattering. J Appl Cryst 2009;42: $347-53$.

[32] Kastengren AL, Powell CF, Arms D, Dufresne EM, Gibson H, Wang J. The 7-BM beamline at the APS: a facility for time-resolved fluid dynamics measurements. J Synchrotron Rad 2012;19:654-57.

[33] Kastengren AL, Tilocco FZ, Duke D, Powell CF, Zhang X, Moon, S. Time-resolved x-ray radiography of sprays from engine combustion network spray A diesel injectors. Atom Sprays 2014;24:251-72.

[34] Jemian PR, Long GG. Silicon photodiode detector for small-angle x-ray scattering. J Appl Cyrst 1990:23:4302.

[35] Zhang, F., J. Ilavsky, G. Long, J. Quintana, A. Allen and P. Jemian. "Glassy Carbon as an Absolute Intensity Calibration Standard for Small-Angle Scattering." Metallurgical and Materials Transactions A 2010:41(5): 11511158.

[36] MacCuspie R, Allen AJ, Nartin MN, Hackley V. Just add water: reproducible singly dispersed silver nanoparticle suspections on-demand. J Nanopart Res 2013:15:1760-8.

[37] Kastengren AL, Tilocco FZ, Powell CF, Manin J, Pickett LM, Payri R, Bazyn T. Engine combustion network (ECN): measurements of nozzle geometry and hydraulic behavior. Atom Sprays 2012;22:1011-52. 
[38] Kastengren AL, Powell CF, Liu Z, Wang J. Time resolved, three dimensional mass distribution of diesel sprays measured with x-ray radiography. SAE Paper 2009-01-0840, 2009.

[39] Pickett LM, Manin J, Kastengren AL, Powell CF. Comparison of near-field structure and growth of a diesel spray using light-based optical microscopy and x-ray radiography. SAE Int J Engines 2014;7:1044-53.

[40] Crua C, Heikal M, Gold M. Microscopic imaging of the initial stage of diesel spray formation. Fuel 2015;157: $1-11$.

[41] Wang TC, Han JS, Xie XB, Lai MC, Henein N, Schwarz E, Bryzik W. Parametric characterization of highpressure diesel fuel injection systems," J Eng Gas Turb Power 2003;125:412-26.

[42] Blaisot J B, Yon J. Droplet size and morphology characterization for dense sprays by image processing: application to the diesel spray. Exp Fluids, 2005;39:977-94.

[43] Zhao H, Liu HF, Cao XK, Li WF, Xu JL. Breakup charcteristics of liquid drops in bag regime by a continuous and uniform air jet flow. Int J Multiphase Flow 2011;37:530-4.

[44] Qian J, Law CK. (1997). Regimes of coalescence and separation in droplet collision. J Fluid Mech 1997;331:59-80. 